\title{
Neutron conduction in the inner crust of a neutron star in the framework of the band theory of solids
}

\author{
N. Chamel ${ }^{1}$ \\ ${ }^{1}$ Institut d'Astronomie et d'Astrophysique, CP-226, \\ Université Libre de Bruxelles, 1050 Brussels, Belgium
}

\begin{abstract}
Even though the "free" neutrons in the inner crust of a neutron star are superfluid, they are still strongly coupled to nuclei due to non-dissipative entrainment effects. These effects have been systematically studied in all regions of the inner crust in the framework of the band theory of solids. Using concepts from solid-state physics, it is shown that the density of conduction neutrons, i.e. neutrons that are effectively "free", can be much smaller than the density of unbound neutrons (by an order of magnitude in some layers) due to Bragg scattering. These results suggest that a revision of the interpretation of various observable neutron-star phenomena may be necessary.
\end{abstract}


Born from the catastrophic gravitational core collapse of massive stars with $M \gtrsim 8 M_{\odot}$ at the end point of their evolution during type II supernova explosions, neutron stars are among the most compact objects in the Universe. The surface of a neutron star, which is composed mainly of iron, is generally obscured by a thin atmosphere. A few meters below the surface at densities above $\sim 10^{4} \mathrm{~g} \cdot \mathrm{cm}^{-3}$, matter is so compressed that atoms, which are arranged in a regular Coulomb lattice, are fully ionized and coexist with a degenerate electron gas. Deeper in the star, nuclei become more and more neutron-rich [1]. At densities above $\sim 4.10^{11} \mathrm{~g} \cdot \mathrm{cm}^{-3}$, some neutrons drip out of "nuclei" thus forming a neutron liquid [2], which is expected to be superfluid at low enough temperatures [3]. The crust of a neutron star extends up to about $\sim 10^{14} \mathrm{~g} \cdot \mathrm{cm}^{-3}$ (i.e. half the density found inside heavy nuclei), at which point nuclei dissolve into a uniform mixture of electrons and nucleons.

Various astrophysical neutron-star phenomena like pulsar glitches or quasiperiodic oscillations in soft-gamma repeaters are expected to be related to the dynamical properties of the neutron superfluid in the inner crust (see e.g. [4] for a recent review). In modeling these events, the neutron superfluid is generally assumed to be flowing freely through the crust because of the absence of viscous drag. This assumption is actually unrealistic. Even though the neutron superfluid can flow without friction, it can still be entrained by the crustal nuclei [5-7]. Unlike viscous drag, this entrainment effect is non-dissipative and therefore it persists in the superfluid phase. Entrainment effects in neutron-star crusts has been already estimated, but only in the shallow layers of the inner crust around the neutron drip density $\sim 4.7 \times 10^{11} \mathrm{~g} \cdot \mathrm{cm}^{-3}$ and near the crust bottom at densities above $5 \times 10^{13} \mathrm{~g} \cdot \mathrm{cm}^{-3}$ using different crust models [8-10]. These results suggested that there might exist regions at intermediate densities where the neutron propagation could be completely suppressed owing to the presence of a band gap in the energy spectrum of unbound neutrons. Such crustal layers would resist the flow of a neutron current in the same way as an ordinary insulator resists the flow of an electric current.

In this paper, entrainment effects are systematically studied in all regions of the inner crust of a cold non-accreting neutron star using a unified treatment based on the nuclear energy density functional theory (EDF), see e.g. [11] for a review. In particuliar, the static long-wavelength neutron current-current correlation function is computed for various densities, ranging from neutron drip to the crust-core transition. This function, which can be expressed in terms of an effective neutron mass or less ambiguously in terms of a density 
of conduction neutrons, is a necessary microscopic ingredient for a realistic hydrodynamical description of the neutron superfluid in the crust [5, 6]. Both the equilibrium composition of the crust and the current-current correlation function have been calculated consistently using the same nuclear energy density functional.

\section{NUCLEAR BAND THEORY OF NEUTRON-STAR CRUSTS}

Since the seminal work of Negele\&Vautherin [12], the inner crust of a neutron star has been generally studied in the framework of the Wigner-Seitz approximation [13] according to which the crust is divided into a set of independent spheres centered around each lattice site. Each cell can thus be seen as an isolated giant "nucleus" for which the usual methods from nuclear physics can be applied. Even though this approach has been fruitful for calculating ground-state properties (at least at not too high densities, as discussed in Refs. [14, 15]), it is inappropriate for studying the low-energy dynamics of "free" neutrons which are delocalized over the whole crust like "free" electrons in ordinary metals. Indeed, the interactions of unbound neutrons with the crystal lattice are highly non-local thus leading to long-range correlations which cannot be properly taken into account in the Wigner-Seitz approximation.

In this work, the neutron conduction in the neutron-star crust will be studied using the band theory of solids [16]. Although this theory has been very successfully employed in various systems in optics, acoustics and condensed matter physics, its application to neutron-star crusts is rather recent [8 10]. The band theory relies on the assumption that the solid can be treated as a perfect crystal. Although the crust of a real neutron star might not be a perfect crystal (see e.g. Section 3.4 of Ref. [4] and references therein), this is still a reasonable approximation for cold non-accreting neutron stars. In the rest frame of the crust, both bound and unbound neutrons are supposed to be described by static periodic mean-fields. In the model we employ here, these fields are generated self-consistently using the nuclear energy density functional theory with a semi-local functional of the Skyrme type [11]. This functional is of the form

$$
E=E_{\mathrm{kin}}+E_{\mathrm{Coul}}+E_{\mathrm{Sky}}
$$

where $E_{\text {kin }}$ is the kinetic energy of the sample volume, $E_{\text {Coul }}$ is the Coulomb energy (using the Kohn-Sham approximation for the exchange part [17]) and $E_{\mathrm{Sky}}=\int \mathrm{d}^{3} \boldsymbol{r} \mathcal{E}_{\mathrm{Sky}}(\boldsymbol{r})$ is the 
nuclear energy. Ignoring pairing, which represents a small correction to the total energy, $E_{\text {Sky }}$ is a functional of the following local densities and currents with $q=n, p$ for neutron, proton respectively: (i) the number density

$$
n_{q}(\boldsymbol{r})=\sum_{\sigma= \pm 1} n_{q}(\boldsymbol{r}, \sigma ; \boldsymbol{r}, \sigma)
$$

(ii) the kinetic density

$$
\tau_{q}(\boldsymbol{r})=\sum_{\sigma= \pm 1} \int \mathrm{d}^{3} \boldsymbol{r}^{\prime} \delta\left(\boldsymbol{r}-\boldsymbol{r}^{\prime}\right) \nabla \cdot \nabla^{\prime} n_{q}\left(\boldsymbol{r}, \sigma ; \boldsymbol{r}^{\prime}, \sigma\right)
$$

and (iii) the spin current vector density

$$
J_{\boldsymbol{q}}(\boldsymbol{r})=-\frac{\mathrm{i}}{2} \sum_{\sigma, \sigma^{\prime}= \pm 1} \int \mathrm{d}^{3} \boldsymbol{r}^{\prime} \delta\left(\boldsymbol{r}-\boldsymbol{r}^{\prime}\right)\left(\boldsymbol{\nabla}-\nabla^{\prime}\right) \times n_{q}\left(\boldsymbol{r}, \sigma ; \boldsymbol{r}^{\prime}, \sigma^{\prime}\right)\left\langle\sigma^{\prime}|\hat{\boldsymbol{\sigma}}| \sigma\right\rangle,
$$

where $n_{q}\left(\boldsymbol{r}, \sigma ; \boldsymbol{r}^{\prime}, \sigma^{\prime}\right)$ is the density matrix in coordinate space (denoting the spin states by $\sigma, \sigma^{\prime}=1,-1$ for spin up, spin down respectively). Introducing the isospin index $t=0,1$ for isoscalar and isovector quantities respectively 1 , the Skyrme functional $E_{\text {Sky }}$ can be expressed as

$$
\mathcal{E}_{\mathrm{Sky}}=\sum_{t=0,1} C_{t}^{n} n_{t}^{2}+C_{t}^{\Delta n} n_{t} \Delta n_{t}+C_{t}^{\tau} n_{t} \tau_{t}+C_{t}^{\nabla J} n_{t} \nabla \cdot \boldsymbol{J}_{\boldsymbol{t}}+\frac{1}{2} C_{t}^{J} \boldsymbol{J}_{\boldsymbol{t}}^{2}
$$

The $C$-coefficients are determined by fitting experimental nuclear data and/or properties of infinite homogeneous nuclear matter as obtained from many-body calculations. The coefficients $C_{t}^{n}$ are generally not constant but depend on the (isoscalar) density $n$. Historically the Skyrme functional was obtained from the Hartree-Fock approximation using zero-range effective nucleon-nucleon interactions of the kind

$$
\begin{aligned}
v_{i, j}= & t_{0}\left(1+x_{0} P_{\sigma}\right) \delta\left(\boldsymbol{r}_{i j}\right)+\frac{1}{2} t_{1}\left(1+x_{1} P_{\sigma}\right) \frac{1}{\hbar^{2}}\left[p_{i j}^{2} \delta\left(\boldsymbol{r}_{i j}\right)+\delta\left(\boldsymbol{r}_{i j}\right) p_{i j}^{2}\right] \\
& +t_{2}\left(1+x_{2} P_{\sigma}\right) \frac{1}{\hbar^{2}} \boldsymbol{p}_{i j} \cdot \delta\left(\boldsymbol{r}_{i j}\right) \boldsymbol{p}_{i j}+\frac{1}{6} t_{3}\left(1+x_{3} P_{\sigma}\right) n(\boldsymbol{r})^{\alpha} \delta\left(\boldsymbol{r}_{i j}\right) \\
& +\frac{\mathrm{i}}{\hbar^{2}} W_{0}\left(\hat{\boldsymbol{\sigma}}_{\boldsymbol{i}}+\hat{\boldsymbol{\sigma}}_{\boldsymbol{j}}\right) \cdot \boldsymbol{p}_{i j} \times \delta\left(\boldsymbol{r}_{i j}\right) \boldsymbol{p}_{i j},
\end{aligned}
$$

where $\boldsymbol{r}_{i j}=\boldsymbol{r}_{i}-\boldsymbol{r}_{j}, \boldsymbol{r}=\left(\boldsymbol{r}_{i}+\boldsymbol{r}_{j}\right) / 2, \boldsymbol{p}_{i j}=-\mathrm{i} \hbar\left(\boldsymbol{\nabla}_{i}-\boldsymbol{\nabla}_{j}\right) / 2$ is the relative momentum, $P_{\sigma}$ is the two-body spin-exchange operator. Minimizing the total energy $E$ for fixed numbers of neutrons and protons leads to the set of self-consistent equations

$$
h_{q}(\boldsymbol{r}) \varphi_{\alpha \boldsymbol{k}}^{(q)}(\boldsymbol{r})=\varepsilon_{\alpha \boldsymbol{k}}^{(q)} \varphi_{\alpha \boldsymbol{k}}^{(q)}(\boldsymbol{r}),
$$

\footnotetext{
${ }^{1}$ Isoscalar quantities, also written without any subscript, are sums over neutrons and protons $\left(\mathrm{e} . \mathrm{g}\right.$. $n_{0}=$ $\left.n=n_{n}+n_{p}\right)$ while isovector quantities are differences between neutrons and protons (e.g. $\left.n_{1}=n_{n}-n_{p}\right)$.
} 
in which the single-particle (s.p.) Hamiltonians $h_{q}(\boldsymbol{r})$ can be expressed as

$$
h_{q}(\boldsymbol{r})=-\nabla \cdot B_{q}(\boldsymbol{r}) \boldsymbol{\nabla}+U_{q}(\boldsymbol{r})-\mathrm{i} \boldsymbol{W}_{\boldsymbol{q}}(\boldsymbol{r}) \cdot \boldsymbol{\nabla} \times \hat{\boldsymbol{\sigma}}
$$

with the various fields defined by

$$
B_{q}(\boldsymbol{r})=\frac{\delta E}{\delta \tau_{q}(\boldsymbol{r})}, U_{q}(\boldsymbol{r})=\frac{\delta E}{\delta n_{q}(\boldsymbol{r})}, \boldsymbol{W}_{\boldsymbol{q}}(\boldsymbol{r})=\frac{\delta E}{\delta \boldsymbol{J}_{\boldsymbol{q}}(\boldsymbol{r})} .
$$

These fields depend on the local densities and currents, Eqs. (2), (3) and (4), which in turn

can be expressed in terms of occupied s.p. wavefunctions $\varphi_{\alpha \boldsymbol{k}}^{(q)}(\boldsymbol{r})$ (see e.g. Ref. [11]). The periodicity of the crystal lattice means that

$$
\begin{gathered}
B_{q}(\boldsymbol{r}+\boldsymbol{\ell})=B_{q}(\boldsymbol{r}), \\
U_{q}(\boldsymbol{r}+\boldsymbol{\ell})=U_{q}(\boldsymbol{r}), \\
W_{\boldsymbol{q}}(\boldsymbol{r}+\boldsymbol{\ell})=W_{\boldsymbol{q}}(\boldsymbol{r}),
\end{gathered}
$$

for any lattice translation vector $\ell$. The boundary conditions to be used in Eqs. (7)-(8) are imposed by the Floquet-Bloch theorem [16]

$$
\varphi_{\alpha \boldsymbol{k}}^{(q)}(\boldsymbol{r}+\boldsymbol{\ell})=\exp (\mathrm{i} \boldsymbol{k} \cdot \boldsymbol{\ell}) \varphi_{\alpha \boldsymbol{k}}^{(q)}(\boldsymbol{r})
$$

where $\boldsymbol{k}$ is the Bloch wave vector and $\alpha$ is the band index.

Because protons are tightly bound to nuclei, proton band structure effects are very small (i.e. the proton s.p. energies are essentially independent of $\boldsymbol{k}$ ) and will not be discussed here. In the following, the neutron energy bands and neutron wave functions will thus be denoted simply as $\varepsilon_{\alpha \boldsymbol{k}}$ and $\varphi_{\alpha \boldsymbol{k}}(\boldsymbol{r})$ respectively.

\section{CONDUCTION NEUTRONS}

The static long-wavelength neutron current-current correlation function determines the mass current of the neutron liquid induced by a change of crystal momentum, the lattice being fixed. Neutron pairing, which gives rise to neutron superfluidity, is expected to have a minor impact on the current-current correlation function [18] and will thus be neglected for simplicity. In the ground state, all neutron s.p. states lying below the Fermi level are occupied so that the neutron liquid is at rest in the crust frame. Considering a small shift 
$\delta \boldsymbol{k}$ in $\boldsymbol{k}$-space of the Fermi surface (FS), each s.p. state will then carry on average a net crystal momentum $\boldsymbol{p}_{\boldsymbol{n}} \equiv \hbar \delta \boldsymbol{k}$ leading to a net mass current $\boldsymbol{j}_{\boldsymbol{n}}$. Neglecting back flow effects (i.e. assuming that the s.p. energies remain unaffected), the current is given to first order in $\delta \boldsymbol{k}$ by

$$
\boldsymbol{j}_{\boldsymbol{n}}=m_{n} \sum_{\alpha} \int \frac{\mathrm{d}^{3} \boldsymbol{k}}{(2 \pi)^{3} \hbar} \delta \tilde{n}_{\alpha \boldsymbol{k}} \nabla_{\boldsymbol{k}} \varepsilon_{\alpha \boldsymbol{k}} \equiv n_{n}^{c} \boldsymbol{p}_{\boldsymbol{n}}
$$

where $m_{n}$ is the neutron mass, $\delta \tilde{n}_{\alpha \boldsymbol{k}} \equiv \tilde{n}_{\alpha \boldsymbol{k}}-\tilde{n}_{\alpha \boldsymbol{k}}^{0}$ denotes the change in the distribution function $\left(\tilde{n}_{\alpha k}^{0}\right.$ being the ground-state distribution) while the density $n_{n}^{\mathrm{c}}$ is defined in terms of the trace of the effective mass tensor introduced in solid-state physics [16]

$$
\begin{gathered}
\left(\frac{1}{m_{n}^{*}(\boldsymbol{k})^{\alpha}}\right)_{i j}=\frac{1}{\hbar^{2}} \frac{\partial^{2} \varepsilon_{\alpha \boldsymbol{k}}}{\partial k_{i} \partial k_{j}}, \\
n_{n}^{\mathrm{c}}=\frac{1}{3} \sum_{\alpha} \int \frac{\mathrm{d}^{3} k}{\left(2 \pi^{3}\right)} \tilde{n}_{\alpha \boldsymbol{k}}^{0} \operatorname{Tr}\left[\frac{m_{n}}{m_{n}^{*}(\boldsymbol{k})^{\alpha}}\right] .
\end{gathered}
$$

Incidentally the neutron effective mass tensor (15) has been also introduced for the study of neutron diffraction in ordinary crystals [19, 20]. Since the ground-state distribution is simply given by $\tilde{n}_{\alpha \boldsymbol{k}}^{0}=H\left(\varepsilon_{\mathrm{F}}-\varepsilon_{\alpha \boldsymbol{k}}\right)$, where $H(x)$ is the Heaviside unit-step distribution, the integral in Eq.(16) has to be taken over the Fermi volume. The Fermi energy $\varepsilon_{\mathrm{F}}$ is the Lagrange multiplier introduced during the minimization of the total energy (11) in order to ensure the conservation of the neutron number

$$
n_{n}=\sum_{\alpha} \int \frac{\mathrm{d}^{3} k}{\left(2 \pi^{3}\right)} \tilde{n}_{\alpha \boldsymbol{k}}^{0}
$$

The density $n_{n}^{c}$ can be interpreted as the density of conduction neutrons by analogy with conduction electrons in ordinary metals. These conduction neutrons are not entrained by nuclei and can thus be considered as being effectively "free". Equation (14) implies that in an arbitrary frame where the crust moves with a velocity $\boldsymbol{v}_{\boldsymbol{p}}$, the neutron mass current will no longer be aligned with the neutron momentum, but will be given by

$$
\boldsymbol{j}_{\boldsymbol{n}}=n_{n}^{c} \boldsymbol{p}_{\boldsymbol{n}}+\left(n_{n}-n_{n}^{c}\right) \boldsymbol{v}_{\boldsymbol{p}}
$$

The quantity $n_{n}-n_{n}^{c}$ can be interpreted as the density of neutrons that are effectively bound to nuclei. 
Alternatively, the neutron conduction can be expressed in terms of an effective mass $m_{n}^{\star}$ by writing the neutron momentum in the crust frame

$$
p_{n} \equiv m_{n}^{\star} \boldsymbol{v}_{\boldsymbol{n}}
$$

where $\boldsymbol{v}_{\boldsymbol{n}}$ is the average velocity of free neutrons defined by

$$
j_{n} \equiv n_{n}^{\mathrm{f}} m_{n} \boldsymbol{v}_{\boldsymbol{n}}
$$

Comparing Eqs. (19) and (20) with (14) leads to the following expression for the effective mass

$$
m_{n}^{\star}=m_{n} \frac{n_{n}^{\mathrm{f}}}{n_{n}^{\mathrm{c}}} .
$$

\section{NUMERICAL RESULTS}

In principle, the ground-state structure of the neutron-star crust at a given average nucleon density could be determined by solving self-consistently the EDF equations (7),(8) and (9) with Bloch boundary conditions (13) under the constraint of beta equilibrium. Considering that the equilibrium structure of the crust is a body centered cubic crystal, these calculations should be repeated for different lattice spacings until the lowest total energy (1) is found. Such calculations would be computionally extremely expensive. For this reason, we have taken the composition of the crust, as found in Ref. [2]. These calculations were based on the fourth-order Extended Thomas-Fermi method with proton shell effects added via the Strutinsky-Integral theorem. Neutron shell effects were neglected since they were shown to be much smaller than proton shell effects [21]. This so-called ETFSI method is actually a high-speed approximation to the self-consistent EDF equations (77), (8) and (9). The functional BSk14 [22] used in Ref. [2] was not only fitted to essentially all the available experimental atomic mass data with a root mean square deviation of $0.73 \mathrm{MeV}$, but was also constrained to reproduce the neutron-matter equation of state of Ref. [23], obtained from many-body calculations using realistic two- and three-body nucleon-nucleon interactions. As a matter of fact, this equation of state is in good agreement with more recent calculations [24-26] in the density domain relevant to neutron-star crusts. For all these reasons, the Skyrme interaction BSk14 is particularly well-suited for studying neutron-star crusts. The neutron and proton density distributions are shown in Fig. 1 for a few crustal layers. 


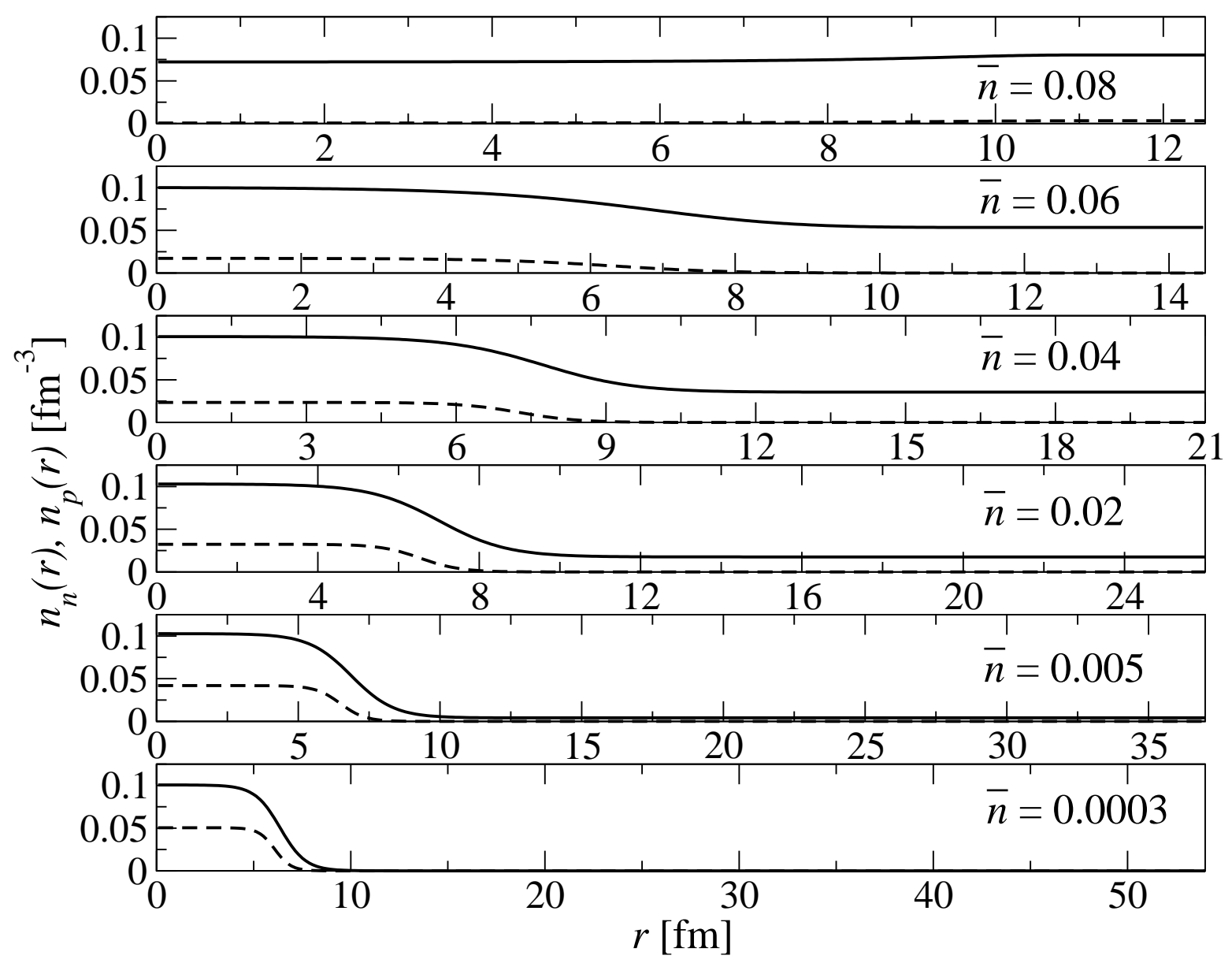

FIG. 1. Neutron (solid line) and proton (dashed line) density profiles inside the Wigner-Seitz cell for different baryon densities $\bar{n}$ (in $\mathrm{fm}^{-3}$ ), as obtained with the ETFSI method [2]. Note the formation of "bubbles" at $\bar{n}=0.08 \mathrm{fm}^{-3}$ : the nucleon densities are slightly larger at the cell edge than at the cell center.

The neutron energy bands $\varepsilon_{\alpha k}$ have been calculated by solving Eqs. (7), (8) and (9) with Bloch boundary conditions (13) using the self-consistent fields obtained in Ref.[2]. The spin-orbit potential $\boldsymbol{W}_{\boldsymbol{n}}(\boldsymbol{r})$, which is proportional to $\boldsymbol{\nabla} n_{n}(\boldsymbol{r})$ and $\boldsymbol{\nabla} n_{p}(\boldsymbol{r})$, is much smaller in neutron-star crusts than that in isolated nuclei and have therefore been neglected. The neutron band structure has been computed by expanding the neutron s.p. wave functions into plane waves

$$
\varphi_{\boldsymbol{k}}(\boldsymbol{r})=\exp (\mathrm{i} k \cdot \boldsymbol{r}) \sum_{\boldsymbol{G}} \widetilde{\varphi_{\boldsymbol{k}}}(\boldsymbol{G}) \exp (\mathrm{i} \boldsymbol{G} \cdot \boldsymbol{r})
$$

where $\boldsymbol{G}$ are reciprocal lattice vectors. By definition, $\exp (\mathrm{i} \boldsymbol{G} \cdot \boldsymbol{\ell})=1$ so that the Bloch boundary conditions (13) are automatically satisfied. In this way, the EDF equation for the 

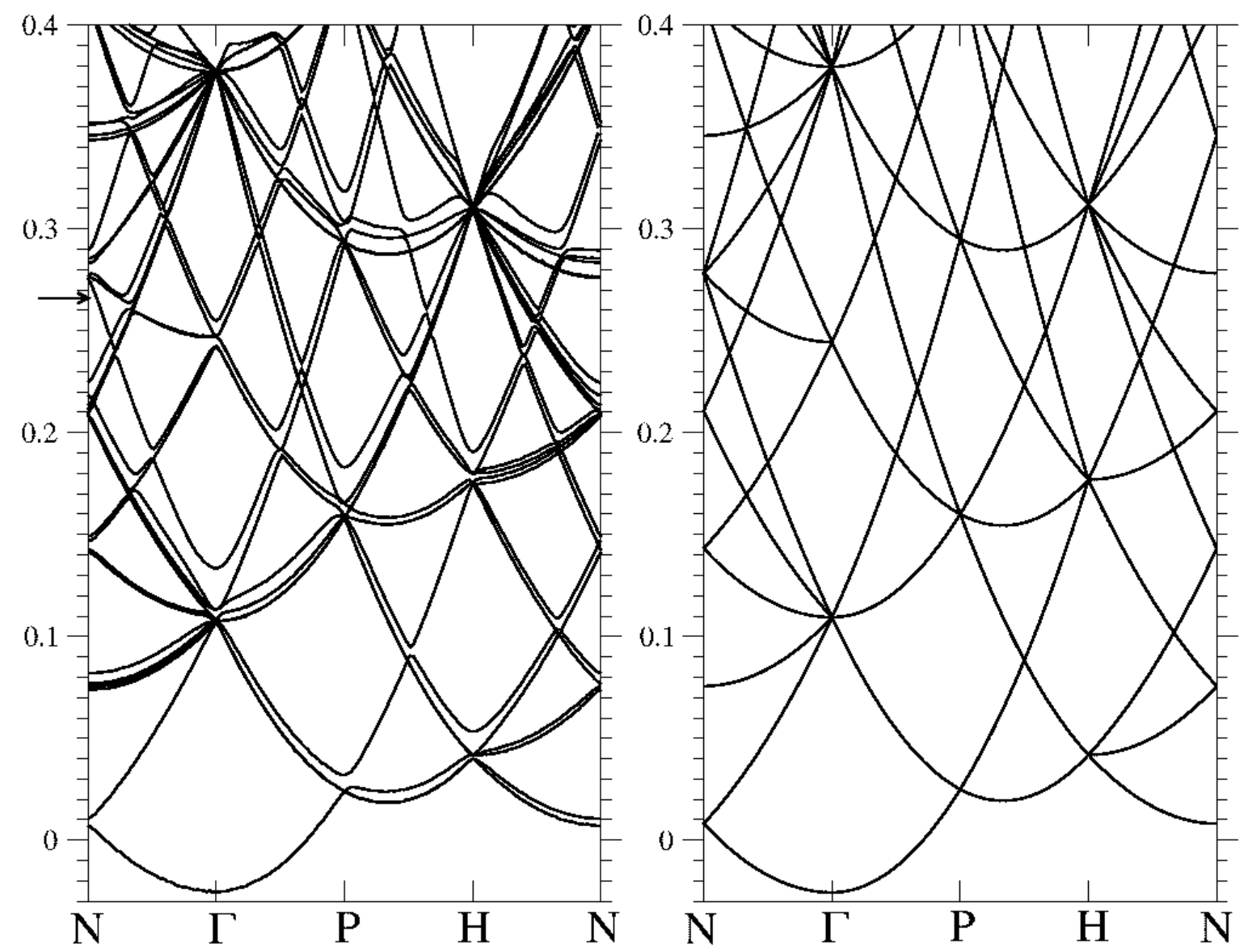

FIG. 2. Left panel: neutron band structure in the inner crust of a neutron star at the average baryon density $\bar{n}=0.0003 \mathrm{fm}^{-3}$ along high-symmetry lines in the first Brillouin zone (only unbound states are shown). The arrow indicates the position of the neutron Fermi energy. Right panel: band structure of a uniform neutron gas at density $n_{n}^{\mathrm{f}}$ (reduced zone scheme). For comparison with the left panel, all bands have been slightly shifted.

neutrons reduces to the matrix eigenvalue problem

$$
\sum_{\beta}\left[\left(\boldsymbol{k}+\boldsymbol{G}_{\alpha}\right) \cdot\left(\boldsymbol{k}+\boldsymbol{G}_{\beta}\right) \widetilde{B_{n}}\left(\boldsymbol{G}_{\beta}-\boldsymbol{G}_{\alpha}\right)+\widetilde{U_{n}}\left(\boldsymbol{G}_{\beta}-\boldsymbol{G}_{\alpha}\right)\right] \widetilde{\varphi}_{\boldsymbol{k}}\left(\boldsymbol{G}_{\beta}\right)=\varepsilon_{\alpha \boldsymbol{k}} \widetilde{\varphi}_{\boldsymbol{k}}\left(\boldsymbol{G}_{\alpha}\right)
$$

with

$$
\widetilde{B_{n}}(\boldsymbol{G})=\frac{1}{V_{\text {cell }}} \int \mathrm{d}^{3} r B_{n}(\boldsymbol{r}) \exp (-\mathrm{i} \boldsymbol{G} \cdot \boldsymbol{r}), \widetilde{U_{n}}(\boldsymbol{G})=\frac{1}{V_{\text {cell }}} \int \mathrm{d}^{3} r U_{n}(\boldsymbol{r}) \exp (-\mathrm{i} \boldsymbol{G} \cdot \boldsymbol{r}) .
$$

These integrals, taken over any primitive cell of volume $V_{\text {cell }}$, can be efficiently calculated using fast Fourier transforms.

In the shallow layers of the inner crust, the linearised augmented plane wave method [10] would have been computionally much faster. Unfortunately this method can be reliably 

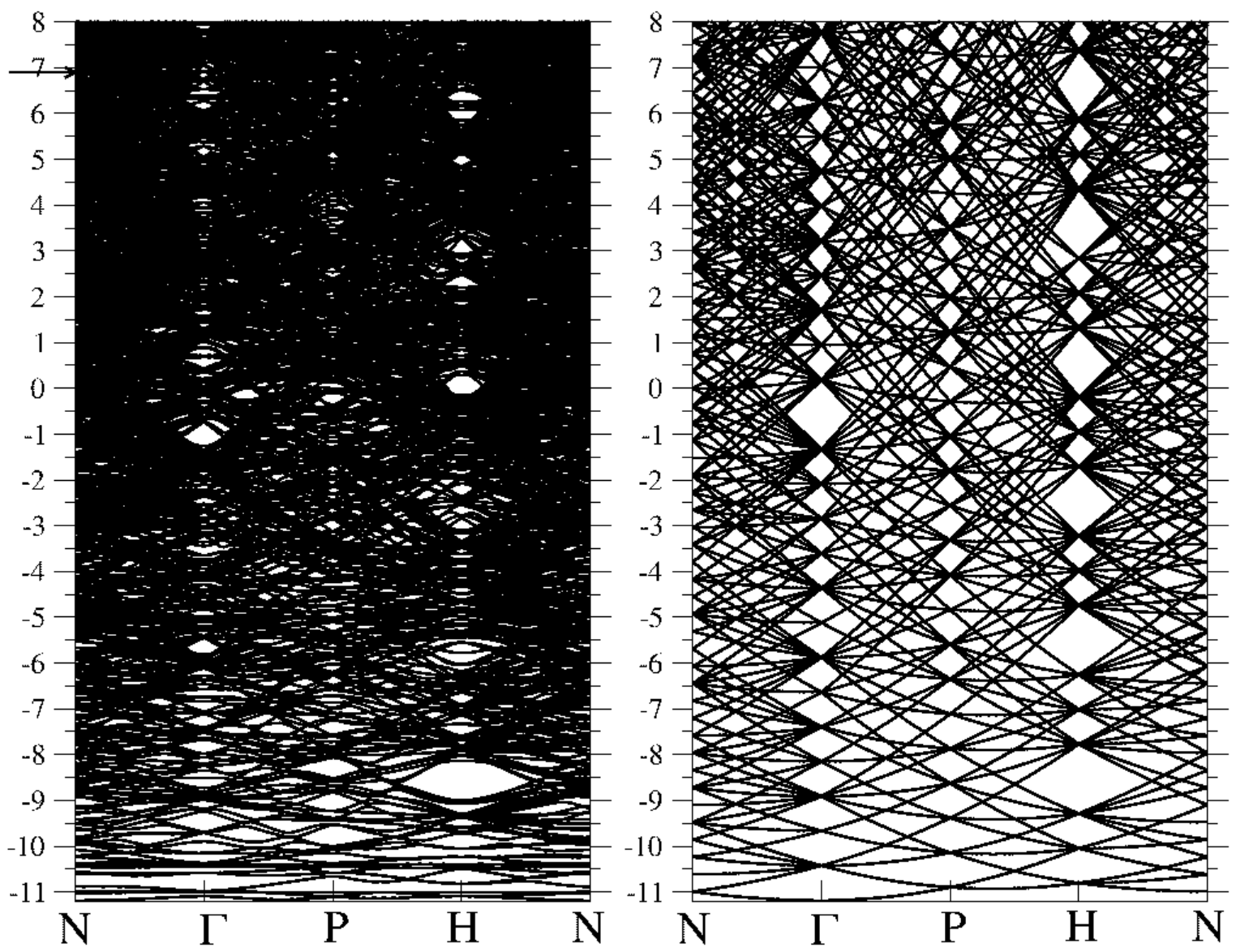

FIG. 3. Left panel: neutron band structure in the inner crust of a neutron star at the average baryon density $\bar{n}=0.03 \mathrm{fm}^{-3}$ along high-symmetry lines in the first Brillouin zone (only unbound states are shown). The arrow indicates the position of the neutron Fermi energy. Right panel: band structure of a uniform neutron gas at density $n_{n}^{\mathrm{f}}$ (reduced zone scheme). For comparison with the left panel, all bands have been slightly shifted.

applied only in the vicinity of the neutron drip transition where only a few neutron bands (in the continuum) are filled. For this reason, the plane wave method has been used in all regions of the inner crust.

The number of calculated neutron bands varies from a hundred in the shallowest layers of the inner crust at $\bar{n}=0.0003$ up to 850 at $\bar{n}=0.03 \mathrm{fm}^{-3}$. A few band structures are shown in Figs 2,3] and 4. We have also shown the band structure in the reduced zone scheme [16] of a uniform neutron gas of density $n_{n}^{\mathrm{f}}$ (empty lattice limit), whose energies are simply given by

$$
\varepsilon_{\alpha \boldsymbol{k}}=B_{n}\left(n_{n}^{\mathrm{f}}\right)\left(\boldsymbol{k}+\boldsymbol{G}_{\alpha}\right)^{2}+U_{n}\left(n_{n}^{\mathrm{f}}\right) .
$$



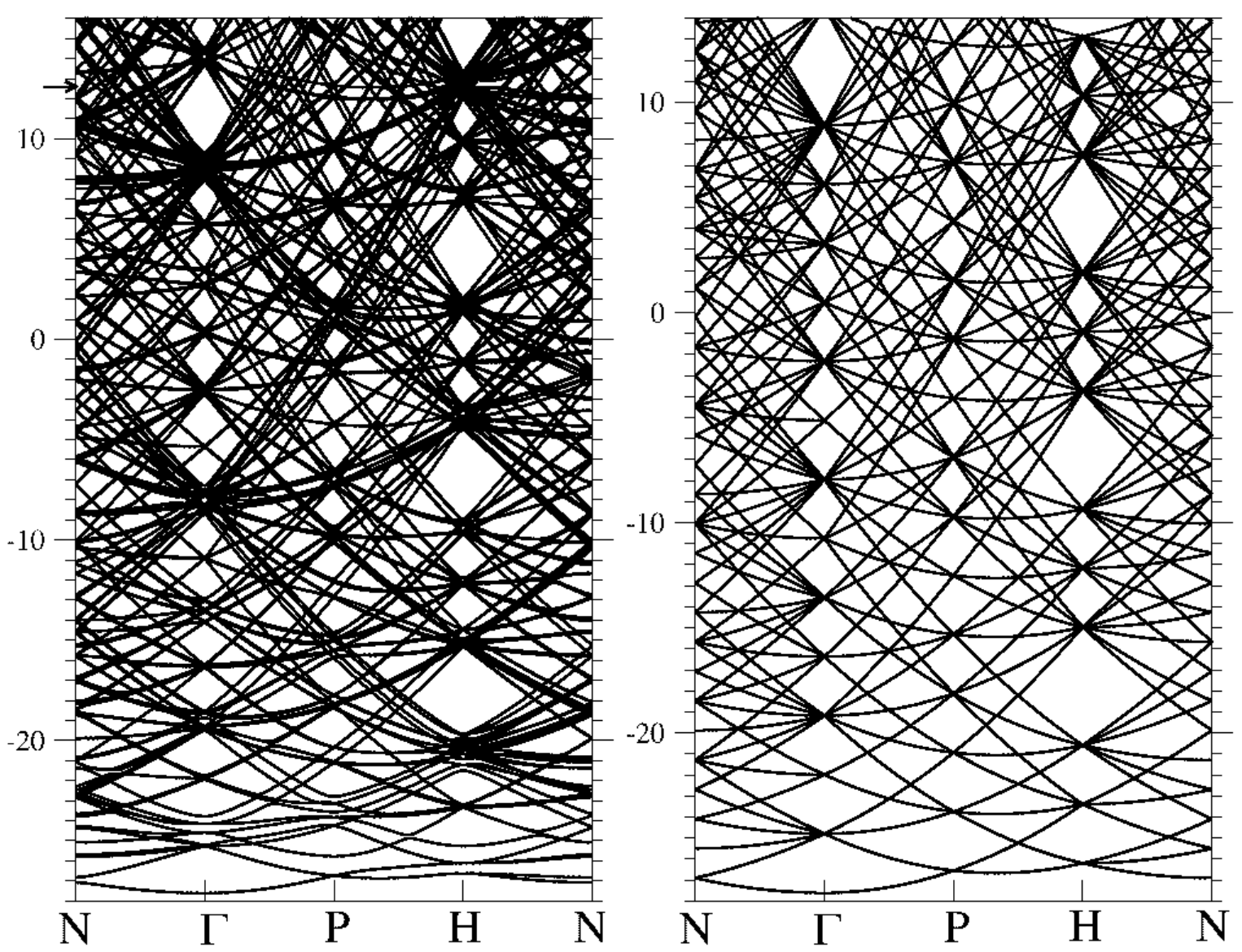

FIG. 4. Left panel: neutron band structure in the inner crust of a neutron star at the average baryon density $\bar{n}=0.08 \mathrm{fm}^{-3}$ along high-symmetry lines in the first Brillouin zone (only unbound states are shown). The arrow indicates the position of the neutron Fermi energy. Right panel: band structure of a uniform neutron gas at density $n_{n}^{\mathrm{f}}$ (reduced zone scheme). For comparison with the left panel, all bands have been slightly shifted.

As can be seen in Fig 2, the energy spectrum of unbound neutron states near the neutron drip point very closely ressembles that of a uniform neutron gas, even though the periodic mean-field potential drops by $\sim 50 \mathrm{MeV}$ inside nuclei. This striking result is the consequence of the Phillips-Kleinman cancellation theorem [27]. The orthogonalization of the unbound states to the bound states leads to an effective repulsive non-local and energy-dependent potential which partially cancels the strongly attractive mean-field potential of nuclei. To a large extent, the same kind of cancellations occurs in the densest regions of the crust as can be inferred from Fig 4. On the contrary, the neutron band structures in the intermediate layers of the inner crust differ significantly from that of a uniform neutron gas, as illustrated in Fig 3. In particular, the energy spectrum is much denser thus revealing that on average 
the energies $\varepsilon_{\alpha \boldsymbol{k}}$ have a much weaker $\boldsymbol{k}$-dependence (almost flat bands) than that given by Eq. (25). As a result, the conduction neutron density $n_{n}^{\mathrm{c}}$ given by Eq. (16) is expected to be much smaller than the density $n_{n}^{\mathrm{f}}$ of unbound neutrons at densities $\bar{n} \sim 0.03 \mathrm{fm}^{-3}$.

The computation of the conduction neutron density requires the evaluation of the effective mass tensor (15) for all occupied bands. In fact, it follows from Eq. (16) and the periodicity of the s.p. energies that completely filled bands do not contribute to the current. This can be easily seen by simply rewriting Eq. (16) as an integral over the Fermi surface using the Green-Ostrogradsky's theorem

$$
n_{n}^{\mathrm{c}}=\frac{m_{n}}{24 \pi^{3} \hbar^{2}} \sum_{\alpha} \int_{\mathrm{F}}\left|\nabla_{\boldsymbol{k}} \varepsilon_{\alpha \boldsymbol{k}}\right| \mathrm{d} \mathcal{S}^{(\alpha)} .
$$

Even though this expression is fully equivalent to Eq. (16), it is computationally much more convenient since only the evaluation of the first derivative of $\varepsilon_{\alpha k}$ is needed. In addition, these derivatives can be easily calculated analytically using the Hellmann-Feynman theorem [28]

$$
\frac{\partial \varepsilon_{\boldsymbol{k}}}{\partial k_{i}}=\sum_{\boldsymbol{G}_{\alpha}, \boldsymbol{G}_{\beta}} \widetilde{\varphi}_{\boldsymbol{k}}\left(\boldsymbol{G}_{\alpha}\right)^{*} \widetilde{B_{n}}\left(\boldsymbol{G}_{\beta}-\boldsymbol{G}_{\alpha}\right)\left(2 k^{i}+G_{\alpha}^{i}+G_{\beta}^{i}\right) \widetilde{\varphi}_{\boldsymbol{k}}\left(\boldsymbol{G}_{\beta}\right)
$$

with the wavefunctions normalized as

$$
\sum_{\beta}\left|\widetilde{\varphi_{k}}\left(G_{\beta}\right)\right|^{2}=1
$$

For each average density $\bar{n}$, the neutron Fermi energy $\varepsilon_{\mathrm{F}}$ has been determined solving Eq. (17) using the mean-value point method [29]. The Fermi surface integral in Eq. (18) has been evaluated with the Gilat-Raubenheimer method [30] using up to 1360 points in the irreducible domain (i.e. 65280 points in the first Brillouin zone) in order to ensure a precision of a few percent. Results are summarized in Table I. As expected from the band structures, the flow of neutrons is almost unaffected by nuclei in the peripheral regions of the inner crust. On the contrary, the neutron conduction is found to be almost completely suppressed at densities $\bar{n} \sim 0.02-0.03 \mathrm{fm}^{-3}$. Whereas more than $90 \%$ of neutrons are unbound in these layers, less than $10 \%$ of them are actually conducting leading to a huge enhancement of the neutron effective mass $m_{n}^{\star} \simeq 13.6 m_{n}$. Incidentally, this result is in close agreement with the effective mass $m_{n}^{s}$ tar $\simeq 15.4 m_{n}$ obtained in a previous work [9] using a different crust model thus suggesting that such strong entrainment effects are generic. However, further work remains to be done exploring the dependence of $m_{n}^{\star}$ on the nuclear energy density functional. 


\begin{tabular}{|c|c|c|c|c|c|}
\hline $\bar{n}\left(\mathrm{fm}^{-3}\right)$ & $\mathrm{Z}$ & $\mathrm{A}$ & $n_{n}^{\mathrm{f}} / n_{n}(\%)$ & $n_{n}^{\mathrm{c}} / n_{n}^{\mathrm{f}}(\%)$ & $m_{n}^{\star} / m_{n}$ \\
\hline 0.0003 & 50 & 200 & 20.0 & 82.6 & 1.21 \\
0.001 & 50 & 460 & 68.6 & 27.3 & 3.66 \\
0.005 & 50 & 1140 & 86.4 & 17.5 & 5.71 \\
0.01 & 40 & 1215 & 88.9 & 15.5 & 6.45 \\
0.02 & 40 & 1485 & 90.3 & 7.37 & 13.6 \\
0.03 & 40 & 1590 & 91.4 & 7.33 & 13.6 \\
0.04 & 40 & 1610 & 88.8 & 10.6 & 9.43 \\
0.05 & 20 & 800 & 91.4 & 30.0 & 3.33 \\
0.06 & 20 & 780 & 91.5 & 45.9 & 2.18 \\
0.07 & 20 & 714 & 92.0 & 64.6 & 1.55 \\
0.08 & 20 & 665 & 104 & 64.8 & 1.54 \\
\hline
\end{tabular}

TABLE I. Composition of the inner crust of cold non-accreting neutron stars as obtained from Ref. 2]. $Z$ and $A$ are respectively the average number of protons and the total average number of nucleons inside the Wigner-Seitz cell. $n_{n}$ is the average neutron density, $n_{n}^{\mathrm{f}}$ is the density of free neutrons as defined by the quantity $\rho_{B n}$ in Ref. [2], $n_{n}^{\mathrm{c}}$ is the density of conduction neutrons and $m_{n}^{\star}$ the neutron effective mass. Note that in the densest layer, $n_{n}^{\mathrm{f}}>n_{n}$ due to the formation of bubbles as indicated in Fig. 1.

\section{MICROSCOPIC ORIGIN OF ENTRAINMENT}

The large discrepancy between the density of unbound neutrons and the density of conducting neutrons is somehow counterintuitive. Indeed in ordinary metals, the electrons that are tighly bound inside the individual atoms constituting the solid have their wavefunction vanishing exponentially outside atoms and are therefore not much affected by the Bloch boundary conditions. As a consequence, their energy bands in $\boldsymbol{k}$-space are essentially flat so that $\nabla_{\boldsymbol{k}} \varepsilon_{\alpha \boldsymbol{k}} \simeq 0$ hence yielding a negligible contribution to the current. The non-trivial electron band structure arises from the most loosely bound "valence" electrons in the isolated atoms which become delocalized in a metal and which can be generally identified with the conduction electrons (still, the density of valence electrons is not exactly equal to the density of conduction electrons). On the contrary, the neutron-saturated "nuclei" found in the inner 
crust of a neutron star only exist because of the Pauli blocking effect from the surrounding neutron liquid but would decay immediately in vacuum. For the reasons mentioned above, neutrons bound inside nuclei do not contribute to the current. Since unbound neutrons are delocalized, one might naively expect that they are all conducting. Indeed, ignoring the crystal lattice and treating the unbound neutrons as a uniform gas of density $n_{n}^{\mathrm{f}}$, it follows immediately from Eq. (16) or Eq. (26) that $n_{n}^{\mathrm{c}}=n_{n}^{\mathrm{f}}$. However it should be emphasized that the density of conduction neutrons is fundamentally different from the density of unbound neutrons: the former characterizes the dynamics of the neutron liquid while the latter is a ground-state property. These two densities are generally not equal because unbound neutrons can be scattered by the crystal according to Bragg's law.

The effects of Bragg scattering are embeddied in the effective mass tensor (15) appearing in the definition (16) of the conduction neutron density. The components of this tensor need not be positive and can actually be negative for wave vectors $\boldsymbol{k}$ such that Bragg reflection is allowed. In particular, an unbound neutron will be reflected whenever its Bloch wave vector $\boldsymbol{k}$ lies on a Bragg plane, i.e. $\boldsymbol{k}$ satisfies the von Laue condition $2 \boldsymbol{k} \cdot \boldsymbol{G}=G^{2}$ for any reciprocal lattice vector $\boldsymbol{G}$. As a result, the neutron current will be suppressed however small the periodic mean-field potential is. Using perturbation theory and setting $B_{n}(\boldsymbol{r}) \equiv B_{n}$ for simplicity, it can be shown that in the vicinity of a Bragg plane the s.p. energies of free neutrons are split into two bands [16]

$$
\varepsilon_{\boldsymbol{k}}^{ \pm} \simeq \frac{1}{2} B_{n}\left(k^{2}+(\boldsymbol{k}-\boldsymbol{G})^{2}\right) \pm \sqrt{\frac{1}{4} B_{n}^{2}\left(k^{2}-(\boldsymbol{k}-\boldsymbol{G})^{2}\right)^{2}+\left|\widetilde{U_{n}}(\boldsymbol{G})\right|^{2}}
$$

It is easily seen that the effect of the periodic potential is to flatten the bands around Bragg planes by introducing a gap of magnitude $2\left|\widetilde{U_{n}}(\boldsymbol{G})\right|$. As a consequence, the conduction neutron density will be reduced whenever the Fermi energy lies on the top (bottom) of the lower (upper) band. The neutron conduction thus depends on two factors: i) the amount of flattening of the bands which in turn is governed by the periodic potential, ii) the position of the Fermi level which is determined by the total neutron density. The more the Fermi sphere intersects Bragg planes, the larger will be the effect of Bragg scattering on the neutron conductivity. The number of intersections depends on the ratio between the Fermi volume and the volume of the first Brillouin zone. According to Luttinger's theorem [31], the Fermi volume associated with unbound states is given by $V_{\mathrm{F}}=(2 \pi)^{3} n_{n}^{\mathrm{f}}$. By definition, the volume of the first Brillouin zone is equal to $V_{\mathrm{BZ}}=(2 \pi)^{3} / V_{\text {cell }}$. Therefore the ratio $V_{\mathrm{F}} / V_{\mathrm{BZ}}=n_{n}^{\mathrm{f}} V_{\text {cell }}$ 
is simply equal to the average number of unbound neutrons inside the Wigner-Seitz cell (or equivalently to the average number of unbound neutrons per nucleus). Basically, this number is the lowest at the neutron drip point, peaks at about 1417 at density $\bar{n}=0.03$ $\mathrm{fm}^{-3}$ and decreases at higher densities. As expected, the resistance of the crust to the flow of a neutron current follows a similar behavior (see Table 【).

\section{CONCLUSIONS}

Despite the absence of viscous drag, the neutron superfluid permeating the inner crust of a neutron star is still strongly coupled to nuclei due to non-dissipative entrainment effects. These effects have been systematically studied in all regions of the inner crust of a cold non-accreting neutron star in the framework of the band theory of solids using an effective Skyrme nuclear energy density functional which not only yields an excellent fit to essentially all experimental atomic masses but was also constrained to reproduce a realistic neutronmatter equation of state.

Some regions of the inner crust are found to strongly resist the flow of a neutron current in the same way as an ordinary insulator resists the flow of an electric current. As a consequence the density of conduction neutrons, i.e. neutrons not entrained by nuclei, turns out to be much smaller than the density of unbound neutrons thus leading to a huge enhancement of the neutron effective mass. These results suggest that a revision of the interpretation of many observable astrophysical phenomena like pulsar glitches [32], quasiperiodic oscillations in Soft-Gamma Repeaters [33] and the cooling of neutron stars [34], may be necessary.

Incidentally, the kind of entrainment effects discussed here are also expected to be observed in laboratory superfluid systems. Indeed, qualitatively similar results have been predicted for a unitary Fermi gas in a 1D optical lattice [35]. Since a dilute neutron gas is approximately in the unitary regime [36], cold atoms experiments could shed light on the properties of superfluid neutrons in neutron-star crusts.

\section{ACKNOWLEDGMENTS}

The author thanks C.J. Pethick and S. Reddy for fruitful discussions. This work was financially supported by FNRS (Belgium) and CompStar, a Research Networking Programme 
of the European Science Foundation. The author thanks the Institute for Nuclear Theory at the University of Washington for its hospitality and the Department of Energy for partial support.

[1] J.M. Pearson, S. Goriely and N. Chamel, Phys. Rev. C83, 065810 (2011).

[2] M. Onsi, A. K. Dutta, H. Chatri, S. Goriely, N. Chamel and J. M. Pearson, Phys. Rev. C 77, 065805 (2008).

[3] N. Chamel, S. Goriely, J.M. Pearson and M. Onsi, Phys. Rev. C 81, 045804 (2010).

[4] N. Chamel and P. Haensel, "Physics of Neutron Star Crusts", Living Rev. Relativity 11, (2008), 10. http://www.livingreviews.org/lrr-2008-10

[5] B. Carter, N. Chamel and P. Haensel, Int. J. Mod. Phys. D 15, 777 (2006).

[6] C.J. Pethick, N. Chamel and S. Reddy, Prog. Theor. Phys. Supplement 186,9 (2010).

[7] V. Cirigliano, S. Reddy, R. Sharma, Phys. Rev. C 84, 045809 (2011).

[8] B. Carter, N. Chamel and P. Haensel, Nucl. Phys. A 748, 675 (2005).

[9] N. Chamel, Nucl.Phys. A747, 109 (2005).

[10] N. Chamel, Nucl. Phys. A773, 263 (2006).

[11] M. Bender, P.-H. Heenen and P.-G. Reinhard, Rev. Mod. Phys.75, 121 (2003).

[12] J.W. Negele and D. Vautherin, Nucl. Phys. A 207, 298 (1973).

[13] E.P. Wigner and F. Seitz, Phys. Rev. 43, 804 (1933) ; Phys. Rev. 46, 509 (1934).

[14] M. Baldo, E.E. Saperstein and S.V. Tolokonnikov, Nucl. Phys. A 775, 235 (2006).

[15] N. Chamel, S. Naimi, E. Khan and J. Margueron, Phys. Rev. C75, 055806 (2007).

[16] N. W. Ashcroft and N. D. Mermin, Solid State physics, Holt, Rinehart and Winston (1976).

[17] W. Kohn and L. J. Sham, Phys. Rev. 1140, 1133 (1965).

[18] B. Carter, N. Chamel and P. Haensel, Nucl. Phys. A 759, 441 (2005).

[19] A. Zeilinger, C. G. Shull, M. A. Horne, and K. D. Finkelstein, Phys. Rev. Lett. 57 (1986), 3089 .

[20] K. Raum, M. Koellner, A. Zeilinger, M. Arif, R. Gahler, Phys. Rev. Lett. 74 (1995), 2859.

[21] K. Oyamatsu and M. Yamada, Nucl. Phys. A578, 181 (1994).

[22] S. Goriely, M. Samyn, J.M. Pearson, Phys. Rev C 75 (2007) 064312.

[23] B. Friedman and V. R. Pandharipande, Nucl. Phys. A361, 502 (1981) 
[24] A. Akmal, V. R. Pandharipande, and D. G. Ravenhall, Phys. Rev. C 58, 1804 (1998).

[25] A. Gezerlis and J. Carlson, Phys. Rev. C 81, 025803 (2010).

[26] K. Hebeler and A. Schwenk, Phys. Rev. C 82, 014314 (2010).

[27] J.C. Phillips and L. Kleinman, Phys. Rev. 59, 287 (1959).

[28] R. Feynman, Phys. Rev. 56,340 (1939).

[29] A. Baldereschi, Phys. Rev. B 7, 5212 (1973).

[30] G. Gilat and L. J. Raubenheimer, Phys. Rev. 144, 390 (1966).

[31] J.M. Luttinger, Phys. Rev. 119, 1153 (1960).

[32] N. Chamel and B. Carter, Mon.Not.Roy.Astron.Soc. 368, 796 (2006).

[33] L. Samuelsson and N. Andersson, Class. Quant. Grav. 26, 155016 (2009).

[34] D. Page and S. Reddy, in "Neutron Star Crust, edited by C. A. Bertulani and J. Piekarewicz, arXiv:1201.5602.

[35] G. Watanabe, G. Orso, F. Dalfovo, L. P. Pitaevskii, S. Stringari, Phys. Rev. A 78, 063619 (2008).

[36] A. Schwenk and C.J. Pethick, Phys. Rev. Lett. 95, 160401 (2005). 